\title{
Pomeron interaction in the perturbative QCD
}

\author{
J.Bartels ${ }^{1}$ and M.A.Braun ${ }^{2}$ \\ ${ }^{1}$ Hamburg University, Germany, \\ ${ }^{2}$ S.Petersburg State University, Russia
}

\begin{abstract}
Formation of the triple pomeron vertices in processes $\mathrm{P} \rightarrow \mathrm{PP} \rightarrow \mathrm{PPP}$ and $\mathrm{PP} \rightarrow \mathrm{P} \rightarrow \mathrm{PP}$ is studied in the BFKL-Bartels approach. It is demonstarted that successive splittings of the pomeron indeed generate the standard triple pomeron vertex in agreement with the dipole approach. In contrast, in the process $\mathrm{PP} \rightarrow \mathrm{P} \rightarrow \mathrm{PP}$ the improper part of the vertex is found missing. Significance of this result is discussed in the framework of the theory of interacting pomerons.
\end{abstract}

\section{Motivation}

In the perturbative QCD strong interactions are mediated by exchange of QCD pomerons ("BFKL pomerons",P), which are bound states of pairs of reggeized gluons ("reggeons") [1]. The latter are extension of the standard gluons to complex angular momenta. Pomerons can interact either by splitting of a pomeron in two $(\mathrm{P} \rightarrow \mathrm{PP})$ or by fusing of a pair of pomerons into one $(\mathrm{PP} \rightarrow \mathrm{P})$. For the splitting the corresponding triple pomeron vertex was constructed both in the BFKL approach $[2,3]$ and dipole picture [4]. It is illustrated in Fig. 1, in which the pomerons coupled to the projectile and targets are shown as ladders of interacting reggeons. In the transverse coordinate space the three participating
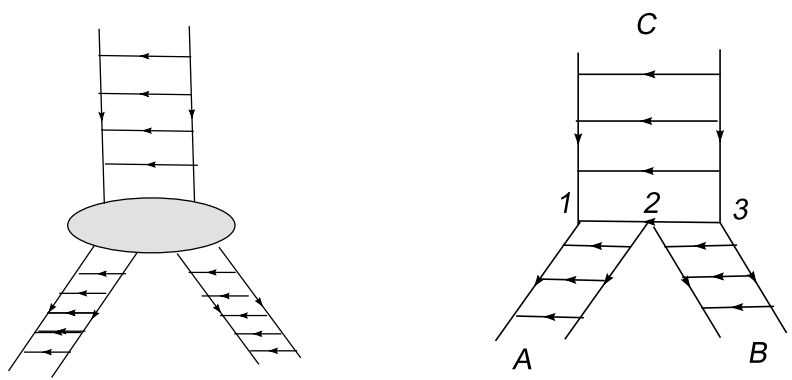

Figure 1. The triple pomeron vertex

pomerons are connected as shown in the right part of Fig. 1. The connecting line passing through 
points $r_{1}, r_{2}$ and $r_{3}$ corresponds to factor [5]

$$
\Gamma\left(r_{1}, r_{2}, r_{3}\right)=-\frac{g^{4} N_{c}}{4 \pi^{3}} \frac{r_{13}^{2} \nabla_{1}^{2} \nabla_{3}^{2}}{r_{12}^{2} r_{23}^{2}}
$$

where $r_{12}=r_{1}-r_{2}$ and so on.

The triple pomeron vertex has played an important role in applications of both the BFKL and dipole approaches to observables. It lies at the origin of the structure function of the heavy nucleus, as given by the Balitski-Kovchegov equation [6,7], which sums pomeron fan diagrams with this vertex (Fig. 2, left). It is also responsible for the pomeron self-mass (Fig. 2, center), which was calculated
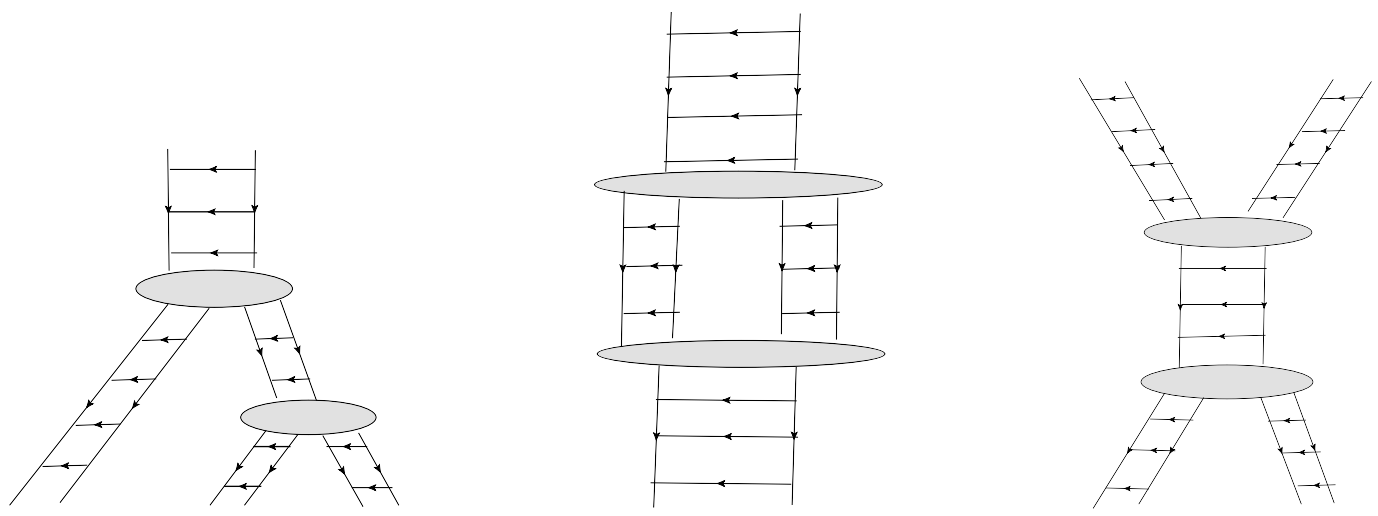

Figure 2. Pomeron fans, self-mass, and transition $\mathrm{PP} \rightarrow \mathrm{P} \rightarrow \mathrm{PP}$

in $[8-11]$.

The splitting and fusing of pomerons by vertex $\Gamma$ was formalized in the effective pomeron action introduced in [12] in which pomerons are described as bilocal fields $\phi\left(r_{1}, r_{2}\right)$ with the interaction Lagrangian

$$
L_{I}=\frac{2 \alpha_{s}^{2} N_{c}}{\pi} \int \frac{d^{2} r_{1} d^{2} r_{2} d^{2} r_{3}}{r_{12}^{2} r_{23}^{2} r_{31}^{2}} \phi\left(r_{1}, r_{2}\right) \phi\left(r_{2}, r_{3}\right) r_{13}^{4} \nabla_{1}^{2} \nabla_{3}^{2} \phi^{\dagger}\left(r_{1}, r_{3}\right)+\text { h.c. . }
$$

As folows from this action in the second order, apart from the self-mass contribution of Fig.2,center, transition $\mathrm{PP} \rightarrow \mathrm{P} \rightarrow \mathrm{PP}$, Fig. 2,right, takes place.

However in the BFKL approach the triple pomeron vertex was derived only for the case of a single splitting of a pomeron in two. Neither two nor more consecutive splittings corresponding to Fig. 2. left and right, have been studied and it was not demonstrated that these processes are governed by two and more vertices $\Gamma$. For fan diagrams Fig. 2, left, there exists an alternative derivation in the dipole picture, which, translated into the BFKL language, indicated that indeed in consecutive splittings exactly vertices $\Gamma$ appear $[13,14]$. However in view of the rather indirect correspondence between the dipole and BFKL approaches it would be desirable to derive this result within the latter approach.

More complicated is the situation with the transition $\mathrm{PP} \rightarrow \mathrm{P} \rightarrow \mathrm{PP}$. In this case the dipole approach cannot be used and the derivation of Fig. 2, right, is a problem.

The point is that within the BFKL approach $\Gamma$ is obtained after summation of transitions of two, three and four reggeons from the colourless short range projectile ( $q \bar{q}$ loop) to the two final pomerons. 
Suppressing evolution in rapidity, which does not influence the triple pomeron vertex, the latter is obtained from the sum of diagrams shown in Fig. 3.
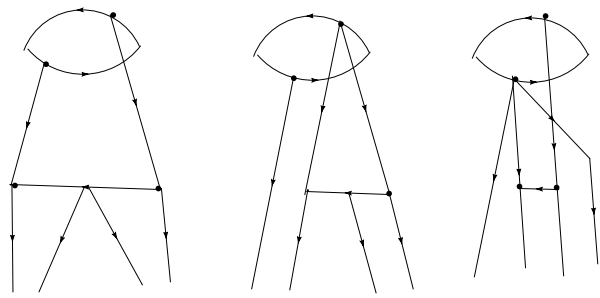

Figure 3. The triple pomeron vertex. Points refer to the coordinate space

Of the three diagrams which sum into $\Gamma$, transitions $\mathrm{P} \rightarrow \mathrm{PP} \rightarrow \mathrm{PPP}$ (Fig. 2, left) and $\mathrm{PP} \rightarrow \mathrm{P} \rightarrow \mathrm{PP}$ (Fig. 2,right) contain only the first.

We demonstrate, in the first place, that additional diagrams for $\mathrm{P} \rightarrow \mathrm{PP} \rightarrow \mathrm{PPP}$ provide the necessary contributions which transform the kernel $K_{2 \rightarrow 4}$ for the second spliting into $\Gamma$ in accordance with the results obtained in the dipole approach. On the other hand, in the transition $\mathrm{PP} \rightarrow \mathrm{P} \rightarrow \mathrm{PP}$ additional diagrams with three and four intermediate reggeons seem to give only a part of the contributions to convert one of the simple kernels $K_{2 \rightarrow 4}$ into $\Gamma$. The rest of the necessary contributions remains missing. In case no new contributions are found, in the transition $\mathrm{PP} \rightarrow \mathrm{P} \rightarrow \mathrm{PP}$ the triple pomeron vertex degenerates into the splitting kernel $K_{2 \rightarrow 4}$, which is in fact one half of the proper part of the standard $\Gamma$.

\section{Three-pomeron vertex in the reggeon approach}

The basis of the derivation of the three-pomeron vertex in the reggeon approach is the so-called bootstrap property of reggeon interaction. It consists in fusing of three and four reggeons in the adjoint colour repersentation into a single reggeon when they are coupled in a special manner to the colourless projectile. This special coupling is provided by a $q \bar{q}$ loop. In this case the amplitudes for coupling of three and four reggeons to the loop, $D_{30}$ and $D_{40}$ are expressed via amplitude $D_{20}$ with only two reggeons coupled to the loop:

$$
\begin{gathered}
D_{30}^{a_{1} a_{2} a_{3}}(1,2,3)=-\frac{1}{2} f^{a_{1} a_{2} a_{3}} g\left(D_{20}(2,31)-D_{20}(1,23)-D_{20}(3,12)\right), \\
D_{40}^{a_{1} a_{2} a_{3} a_{4}}(1,2,3,4)=-d^{a_{1} a_{2} a_{3} a_{4}} D_{40}^{1234}(1,2,3,4)-d^{a_{2} a_{1} a_{3} a_{4}} D_{40}^{2134}(1,2,3,4),
\end{gathered}
$$

where

$$
\begin{gathered}
D_{40}^{1234}=g^{2}\left(D_{20}(1,234)+D_{20}(123,4)-D_{20}(14,23)\right) \\
D_{40}^{2134}=g^{2}\left(D_{20}(2,134)+D_{20}(124,3)-D_{20}(12,34)-D_{20}(13,24)\right)
\end{gathered}
$$

for two states of 4 reggeons, which differ in their order on the surface of a cylinder (indicated by the upper index). Here 12 means $q_{1}+q_{2}, a_{i}$ are colour indices and $d^{a_{1} a_{2} a_{3} a_{4}}=\operatorname{Tr}\left(t_{1}^{a} t_{2}^{a} t_{3}^{a} t_{4}^{a}+t_{4}^{a} t_{3}^{a} t_{2}^{a} t_{1}^{a}\right)$. The bootstrap ensures that evolution does not change these relations, that is the full amplitudes $D_{3}$ and $D_{4}$ for transition from the loop to 3 and 4 reggeons with all BFKL interactions and transitions from 2 to 3 and from 2 to 4 reggeons is given by the same formulas Eqs. (3), (5) and (6) with $D_{20}$ substituted 
by the BFKL pomeron $D_{2}$ ('reggeization') As a result transitions to 2 final pomerons all turn out to start also from the pomeron state and so can be expressed via the triple pomeron vertex. In fact the equation for this amplitude has a structure of a Shroedinger equation in rapidity and transverse momenta with certain inhomogeneous terms, which apart from the direct interaction with the $q \bar{q}$ loop, represent transitions into the two-pomeron state from two, three and four evolved reggeons coming from the loop. The three-pomeron vertex can be found from these latter inhomogeneous terms

$$
D_{2 \rightarrow 2 P}^{(0)}+D_{3 \rightarrow 2 P}^{(0)}+D_{4 \rightarrow 2 P}^{(0)}=\Gamma D_{20}+R_{2 P}^{(6)},
$$

where $R_{2 P}^{(6)}$ is the reggeized $D_{40}$ in order $g^{6}$.

It is essential that this result does not use the explicit form of function $D_{20}(1,2)$ and is valid if one substitutes $D_{20}$ with arbitrary function $F_{2}(1,2)$ which vanishes when $q_{1}$ or $q_{2}$ vanishes. It is only important that the impact factors $F_{3}$ and $F_{4}$ for 3 and 4 reggeons are related to $F_{2}$ in the same way as $D_{30}$ and $D_{40}$ to $D_{20}$.

\section{Pomeron fan diagrams}

In the fan diagrams (Fig. 2,left) in the first splitting all contributions from Fig. 3 take part, so that the standard vertex $\Gamma$ is formed. However already in the second splitting there seems to enter only the first contribution. However in fact one finds two additional diagrams in which instead of the first splitting into 4 reggeons there appear splittings into 5 and 6 reggeons, shown in Fig. 4.
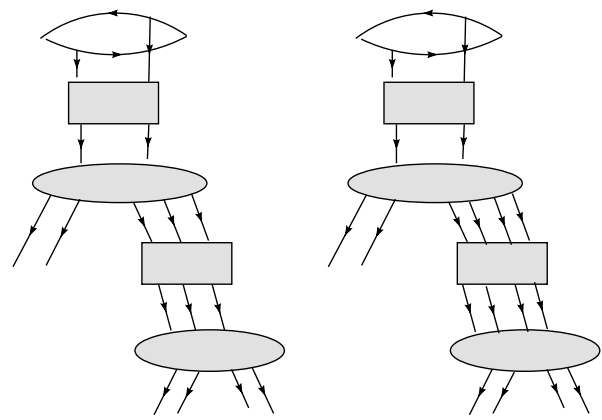

Figure 4. Additional pomeron fan diagrams to be added to Fig.2, left

As we shall demonstrate they convert vertex $K_{2 \rightarrow 3}$ to the standard vertex $\Gamma$.

\section{4 reggeons after the first splitting}

After the first splitting we have the general 4-reggeon aplitude $D_{4}$. Its part corresponding to the final state of two pomeron states made of reggeons 1,2 and 3,4 is given by $D_{4}^{a_{1} a_{2} a_{3} a_{4}}=$ $\delta_{a_{1} a_{2}} \delta_{a_{3} a_{4}} D_{4}(1,2,3,4)$. Amplitude $D_{4}(1,2,3,4)$ can be expresed via function

$$
G(1,2,3)=K_{3 \leftarrow 2}(1,2,3) D_{2}+D_{2}(1,23)(\omega(2)-\omega(23))+D_{2}(12,3)(\omega(2)-\omega(12)),
$$

where $K_{2 \rightarrow 3}$ describes transition from 2 to 3 reggeons and $\omega$ is the reggeon trajectory.

$$
D_{4}(1,2,3,4)=\frac{1}{2} g^{2}\{G(1,23,4)+G(2,13,4)+G(1,24,3)+G(2,14,3)\}
$$


Coupling to the pomeron $P(1,2)$ we 0 btain $g^{2}(G(1,23,4)+G(1,24,3)) \otimes P(1,2)$. Here $\otimes$ means integration over the pair of intermediate momenta. Introducing function $f(3,4)=G(1,23,4) \otimes g^{2} P(1,2)$ we find that before the second splitting the final 4-gluon amplitude is coupled to a symmetric function $F_{2}(3,4)=f(3,4)+f(4,3)$. It takes the role of the loop $D_{20}$ for the second splitting.

\section{5 reggeons after the first splitting}

Here before the second splitting we find amplitude $D_{5}$ [17] for transition of the initial pomeron into five outgoing reggeons, two of which 1 and 2 are to transform into the outgoing pomeron and the remaining three, 3,4 and 5, serve as ingoing for the second splitting. $D_{5}$ contains 10 terms, all are expressed via $D_{4}$. Only three terms contribute to our colour structure. The first of them can be written in terms of fuction $G$ as follows

$$
(1)=\delta_{a_{1} a_{2}} f^{a_{3} a_{4} a_{5}} \frac{1}{2} g^{2} N_{c}^{2}\{G(1,234,5)+G(2,134,5)+G(1,25,34)+G(2,15,34)\} .
$$

Coupling 1 and 2 to the outgoing pomeron we get $(1) \otimes P(1,2)=f^{a_{3} a_{4} a_{5}} F(34,5)$, where $F$ was defined above.

The remaining two terms give results corresponding to interchanges $(4 \leftrightarrow 5)$ and $(3 \leftrightarrow 4)$ and in this way will add terms corresponding to the full interaction with the $q \bar{q}$ loop: $(2) \otimes P(1,2)=$ $-f^{a_{3} a_{4} a_{5}}\left(F(35,4)\right.$ and $(3) \otimes P(1,2)=f^{a_{3} a_{4} a_{5}} F(45,3)$.

In the sum we get the impact factor for transition to 3 reggeons 3,4 and 5

$$
F_{3}=\frac{1}{2} g f^{a_{3} a_{4} a_{5}}((1)+(2)+(3))=\frac{1}{2} g f^{a_{3} a_{4} a_{5}}(F(34,5)+F(45,3)-F(35,4))
$$

exactly folowing the form (3).

\section{6 gluons after the first splitting}

The corresponding amplitude $D_{6}$ satisfies the Shroedinger equation with 6 inhomogeneous terms [17]. Only two of them give a non-zero contribution in our colour configuration: $T_{1}=$ $\sum d^{a_{1} a_{2} a_{3} a_{4}} \delta_{a_{5} a_{6}} I(1,2,3,4 \mid 5,6)$ and $T_{2}=\sum d^{a_{2} a_{1} a_{3} a_{4}} \delta_{a_{5} a_{6}} J(1,2,3,4 \mid 5,6)$. The sums go over all partitions of reggeons into two groups with 4 reggeons in one of them and 2 in the other.

We start with $T_{1}$. The relevant term from it is $T_{1}=\delta_{a_{1} a_{2}} d^{a_{3} a_{4} a_{5} a_{6}} I(3,4,5,6,1,2)$. Here according to $[17]$

$$
I(3,4,5,6 \mid 1,2)=-g^{2}\left(D_{4}(3,456,1,2)+D_{4}(345,6,1,2)-D_{4}(36,45,1,2)\right) .
$$

Via function $G$.

$$
\begin{array}{r}
D_{4}(3,456,1,2)=\frac{1}{2} g^{2}[G(3,456,2)+G(456,13,2)+G(3,456,1)+G(456,32,1)], \\
D_{4}(345,6,1,2)=\frac{1}{2} g^{2}[G(345,16,2)+G(6,345,2)+G(345,26,1)+G(6,345,1)], \\
D_{4}(36,45,1,2)=\frac{1}{2} g^{2}[G(36,451,2)+G(45,361,2)+G(36,452,1)+G(45,362,1)] .
\end{array}
$$

Convolution with the pomeron $P(1,2)$ gives

$$
T_{3} \otimes P(1,2)=-g^{2} d^{a_{3} a_{4} a_{5} a_{6}}\left(F_{2}(456,3)+F_{2}(6,345)-F_{2}(36,45)\right) .
$$

Comparing with (5) we observe that it is the correct structure for $D_{40}$. for the order of gluons 1234 .

Now $T_{2}$. The relevant term from it is $T_{2}=\delta_{a_{1} a_{2}} d^{a_{4} a_{3} a_{5} a_{6}} J(4.3,5,6,1,2)$ where from [17]

$$
J(4,3,5,6 \mid 2,)=-g^{2}\left(D_{4}(456,3,2,1)+D_{4}(346,5,2,1)-D_{4}(34,56,2,1)-D_{4}(45,36,2,1)\right) .
$$


In terms of $G$

$$
\begin{aligned}
& D_{4}(456,3,2,1)=\frac{1}{2} g^{2}[G(456,32,1)+G(42,356,1)+G(456,31,2)+G(41,356,2)], \\
& D_{4}(346,5,2,1)=\frac{1}{2} g^{2}[G(346,52,1)+G(52,346,1)+G(346,51,2)+G(51,346,2)], \\
& D_{4}(34,56,2,1)=\frac{1}{2} g^{2}[G(34,562,1)+G(56,342,1)+G(34,561,2)+G(56,341,2)], \\
& D_{4}(45,36,2,1)=\frac{1}{2} g^{2}[G(45,362,1)+G(36,452,1)+G(45,361,2)+G(36,451,2)] .
\end{aligned}
$$

Convoluting with pomeron $P(1,2)$ we find

$$
T_{4} \otimes P(1,2)=-g^{2} d^{a_{4} a_{3} a_{5} a_{6}}\left[F_{2}(4,356)+F_{2}(5,345)-F_{2}(35,46)-F_{2}(34,56)\right] .
$$

This has the same structure as the term $D_{40}$ for the order or reggeons 2134 ., Eq, (6).

So collecting our results we see that the initial states for the second evolution into 4 reggeons 3456 are exactly the same as for the evolution into 4 reggeons in Fig. 3 with the only difference that loops $D_{20}$ are substituted by functions $F_{2}$. T As a result due to Eq. (7) the second splitting in Fig. 2,left, will be accomplished by the same vertex $\Gamma$ as the first splitting, in accordance with the results from the dipole picture.

\section{Transitions $\mathrm{PP} \rightarrow \mathrm{P} \rightarrow \mathrm{PP}$}

In this section we discuss the possibility to obtain the structure shown in Fig. 2,right, in which two initial pomerons go into the intermediate pomeron and then into two final pomerons via the vertex $\Gamma$. As stressed in the introduction, vertex $\Gamma$ is local in rapidity, has a fixed order $g^{8}$ and does not depend on $y$. So, to locate it in diagrams, we can neglect evolution in rapidity and study diagrams of order $g^{8}$ apart from the $g$-dependence of impact factors. This means that our aim reduces to study if we can find diagrams of order $g^{8}$ which, added to the contribution of the diagram in Fig. 5,A, transform it into the diagram in Fig. 5,B.

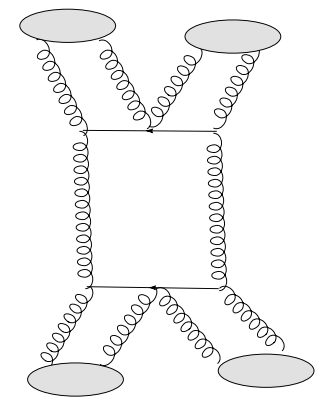

A

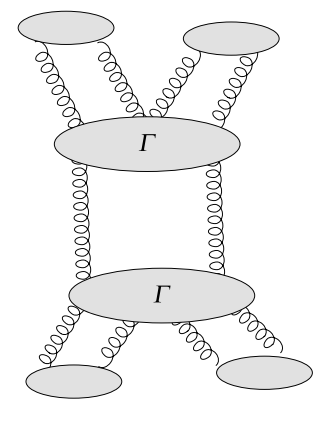

$B$

Figure 5. $\mathrm{PP} \rightarrow \mathrm{P} \rightarrow \mathrm{PP}$ with two $K_{2 \rightarrow 4}(\mathrm{~A})$ and with two $\Gamma$ (B) in the lowest order

All typical diagrams in this order are illustrated in Fig. 6. Apart from the trivial term with two 

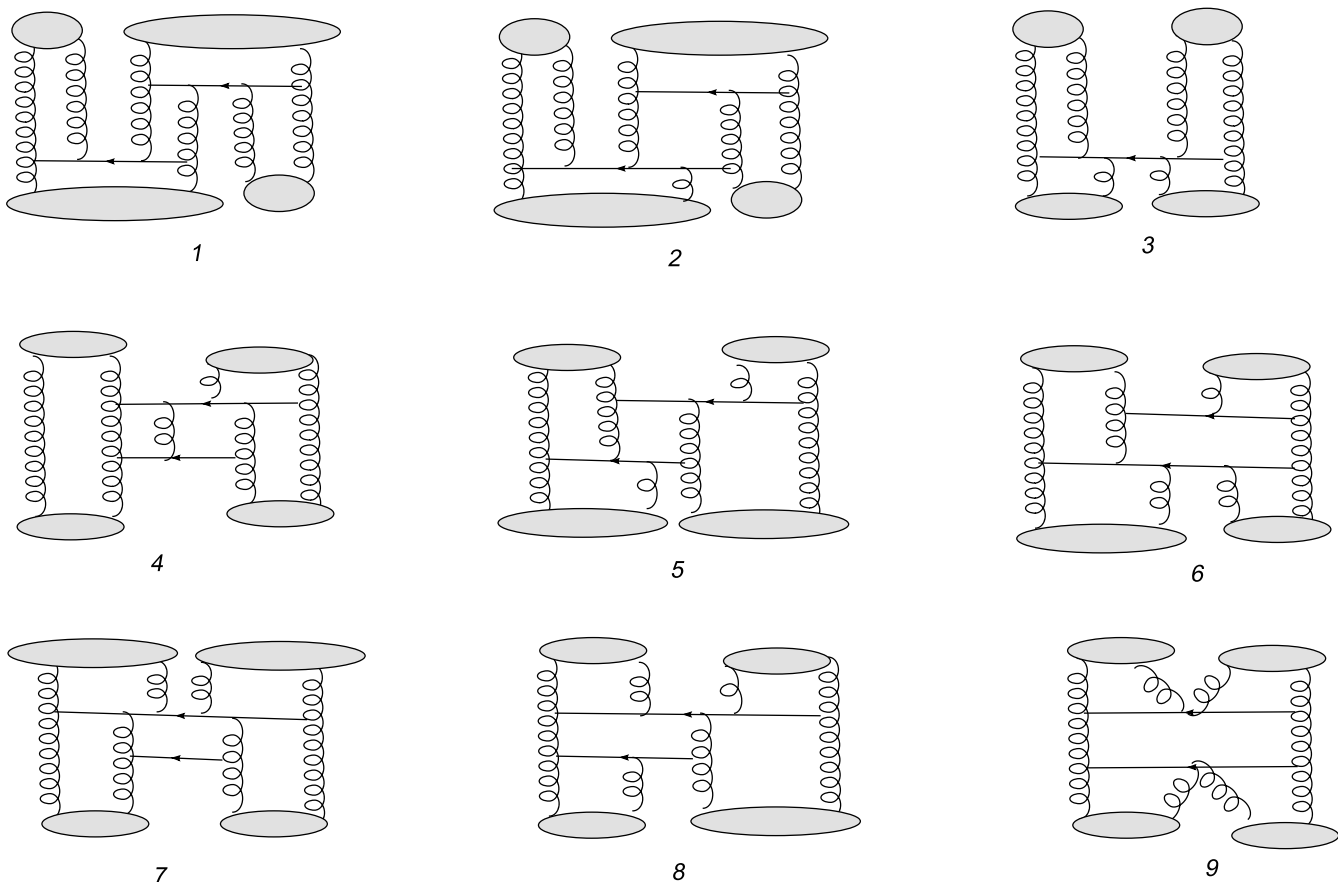

Figure 6. Diagrams of order $g^{8}$

kernels $K_{2 \rightarrow 4}$ posible candidates for the necessary additional contributions are reduced to diagrams 7, 8 and similar ones with the targets and projectile exchanged (3 and 6). Note that after convolution with, say, the initial pomerons these terms are to depend on only one variable.

\section{Diagram in Fig.6,8}

The diagram in Fig. 6,8 contains the kernel $K_{3 \rightarrow 4}$ [18]. It is composed of three parts shown graphically in Fig. 7. . After convolution of $K_{3 \rightarrow 4}$ with the upper impact factors we find one term in

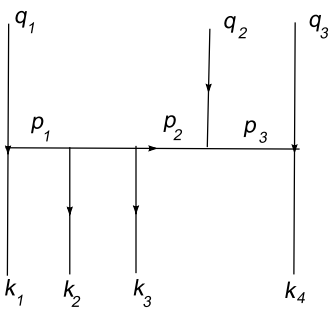

1

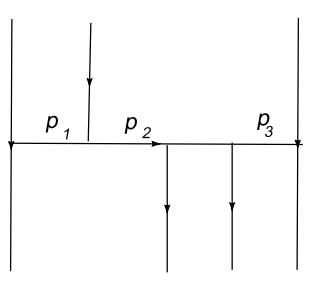

2

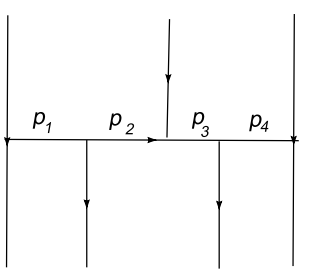

3

Figure 7. Kernel $K_{3 \rightarrow 4}$ 
part 1 and another one in part 2 which give the necessary structure $g\left(F_{2}\left(q_{1}, q_{23}\right)+F_{2}\left(q_{3}, q_{12}\right)\right) / 2$. So contribitions from 3 intermediate reggeons give two of the necessary contributions to $F_{3}$ but the third term $-g F_{2}\left(q_{2}, q_{13}\right) / 2$ is missing.

\section{Diagram in Fig.6,7}

The diagram in Fig. 6,7 contains kernel $K_{4 \rightarrow 4}$ It is composed of six parts shown graphically in Fig. 8. . Four of them 1,2,3 and 6 can be expressed via the already known 3 parts of $K_{3 \rightarrow 4}$. parts 4
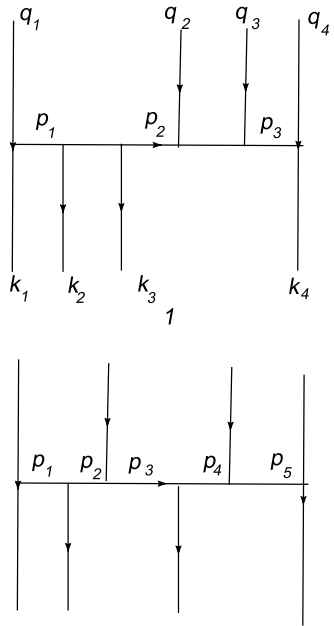

4

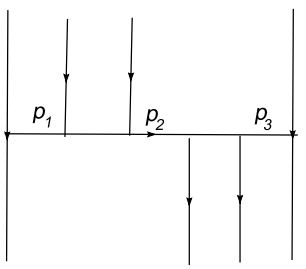

2

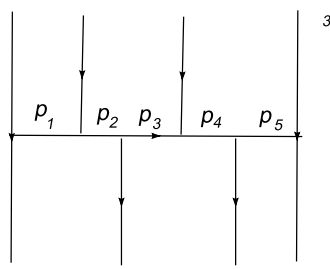

5

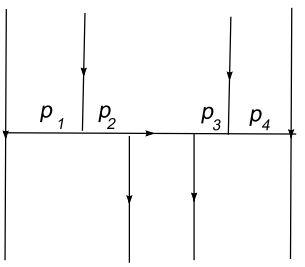

3

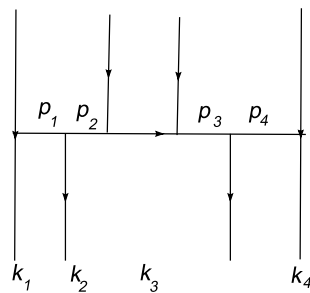

6

Figure 8. Kernel $K_{4 \rightarrow 4}$

and 5 are new. From parts one and two we find contributions $-g^{2} d^{a_{1} a_{2} a_{3} a_{4}}\left(F_{2}\left(q_{1}, q_{234}\right)+F_{2}\left(q_{4}, q_{123}\right)\right)$.

From part 3 we find a term with the correct momentum structure $C_{3} F_{2}\left(q_{12}, q_{34}\right)$. but with the wrong color factor $C_{3}=\left(d^{a_{1} a_{2} a_{3} a_{4}}-d^{a_{2} a_{1} a_{3} a_{4}}\right) / 2$ which does not correspond to (6) and violates the bootstrap.

No more terms depending on only one variable appear.

So we conclude that diagram Fig. 6,7 indeed gives two of the terms in (4). However the rest 5 terms are missing.

\section{Conclusion}

We have found that in successive splittings of the pomeron (fan diagrams) new diagrams with production of extra reggeons and higher splitting kernels $K_{2 \rightarrow n}$ with $n=5$ and 6 provide the contributions which are needed to form the three-pomeron vertex at each splitting. This result agrees with conclusions in the framework of the dipole model, as expressed in the Balitski-Kovchegov equation.

In contrast, we have not been able to discover contributions needed to form two three-pomeron vertices in the transition $\mathrm{PP} \rightarrow \mathrm{P} \rightarrow \mathrm{PP}$. A part of these contribution necessary to form one of the vertices was found from diagrams generated by kernels $K_{3 \rightarrow 4}$ and $K_{4 \rightarrow 4}$ but the rest is missing. So even the possibility to form one of the vertices $\Gamma$ is questionable. If the necessary contributions are indeed absent then the vertex connecting the two initial and two final pomerons with the intermediate one reduces to just the simple kernel $K_{2 \rightarrow 2}$, that is to one half of the proper part of $\Gamma$. 


\section{References}

[1] V.S.Fadin, E.A.Kuraev, L.N.Lipatov, Phys. lett. B 60 (1975) 50; Ya.Ya. Balitsky, L.N.Lipatov, Sov. J. Nucl. Phys., 28 (1978) 822

[2] J.Bartels, Z.Phys. C 60 (1993) 471

[3] J.Bartels and M.Wuesthoff, Z.Phys. C 66 (1995) 157

[4] A.H.Mueller, Nucl. Phys. B 415 (1994) 373; B 437 (1995) 107; A.H.Mueller and B.Patel, Nucl. Phys. B 425 (1994) 471

[5] M.A. Braun and G.P.Vacca, Eur. Phys. J. C 6 (1997) 147

[6] I.Balitski, Nucl. Phys. B 463 (1996) 99

[7] Yu.V. Kovchegov, Phys.Rev D 60 (1999) 034008

[8] J.Bartels, M.Ryskin and G.P.Vacca, Eur. Phys. J. C 27 (2003) 101

[9] R. Peshanski. Phys. Lett. B 409 (1997) 491

[10] M.A.Braun, Eur. Phys. J. C 63 (2009) 287

[11] M.A.Braun and A.N. Tarasov, arXiv: 1304.3014

[12] M.A.Braun, Eur. Phys. J. 48 (2006) 511

[13] M.A.Braun, Eur.Phys.J. c 16 (2000) 337

[14] J.Bartels, L.N.Lipatov, G.P.Vacca, Nucl. Phys. B 706 (2005) 391

[15] J.Bartels, Nucl. Phys. B 175 (1980) 365; J. Kwiecinski, M.Praszaloviicz, Phys. Lett. B 94 (1980) 413

[16] M.A.Braun, Eur. Phys. J. C 6 (1999) 321

[17] J.Bartels and C.Ewerz, JHEP 9909 (1999) 026

[18] J.Bartels, Nucl. Phys. B 175 (1980) 365 